\title{
Role of Renal Arterial Embolization (Rae) In Treatment of Iatrogenic Renal Arterial Injuries
}

\author{
Ahmed Fathy Abd EL Ghany, Marwa ElSayed Abd El Rahman, Dr.Karim Ahmed Abd El Tawab, \\ Zeinab Aly Moussa Aly \\ Radiology Department, Faculty of Medicine, Ain Shams University \\ zeinabalymoussa26@gmail.com
}

\begin{abstract}
Background: Trans-catheter renal arterial embolization (RAE) has emerged as a possible alternative to surgery in the management of iatrogenic renal arterial injuries. Objective: To discuss the role of renal arterial embolization in patients with iatrogenically-induced renal arterial injuries.

Methodology: All cases were done at the Interventional Radiology Unit, Ain Shams University Hospital.

Results: Technical and Clinical success rates of the RAE reached $90 \%$ for each, with post procedural complications that only amounted up to $30 \%$, half of which was the post embolization syndrome that presents as fever, leukocytosis and pain and is treated conservatively.

Conclusion: Renal artery embolization has proven to be a safe, minimally invasive option in the treatment of iatrogenic renal arterial injuries achieving high technical and clinical success rates.
\end{abstract}

Keywords: Renal arterial injuries, Renal artery embolization (RAE), Renal Pseudo-aneurysm, Renal arteriovenous fistula (AVF).

\section{INTRODUCTION}

Renal arterial injuries could develop following blunt trauma as in road traffic accidents, penetrating trauma as in stab wounds or iatrogenically post urological interventions: renal surgeries, percutaneous nephrolithotomy (PCNL), renal trucut biopsy and percutaneous nephrostomy tube application. Life threatening hemorrhagic complications could occur in up to $15 \%$ of those patients ${ }^{(\mathbf{1})}$.

Surgical corrections of bleeders could exacerbate the condition by releasing the tamponade effect of the perirenal hematoma, as well as higher postoperative morbidity/mortality and longer hospital stay ${ }^{(2)}$.

Trans-catheter arterial embolization is an endovascular approach devised to control the post traumatic renal hemorrhage. It has recently emerged as an alternative to operative correction of renal arterial compromise since it is minimally invasive, with fewer postoperative complications and shorter hospital stay ${ }^{(4)}$.

The role of trans-catheter renal artery embolization has presently become more renouned due to the great advancement in the development of embolizing agents as : (vascular coils, particles and endovascular glue) as well as the introduction of the microcatheters enabling superselective bleeder localization and catheterization with minimal parenchymal loss ${ }^{(\boldsymbol{6})}$.

The choice of embolic agents depends upon: the site of the bleeder whether proximal or distal, size of the injured artery, hemodynamic status of the patient, accessibility of the source of bleeding and ability to spare other non-bleeding arteries for the preservation of the normal renal parenchyma ${ }^{(\mathbf{8})}$.

Coils are used for proximal large bleeders embolization. Polyvinyl alcohol (PVA) particles are used for small distal bleeders. Glue is used for hemodynamically unstable patients with an abnormal bleeding profile ${ }^{(9)}$.

Little is known about the side effects of the renal arterial embolization, which might result from over devascularization of a large portion of renal parenchyma or renin secretion by the ischemic renal parenchyma followed by hypertension development ${ }^{(\mathbf{1 0})}$.

\section{AIM OF WORK}

The aim of this study was to assess the success rates of the technical methodology and the short term clinical outcome of RAE in patients with iatrogenic renal arterial injuries. Investigation and treatment of the cases were done at Ain Shams University Hospitals.

\section{PATIENTS AND METHODS}

\section{Patients}

During a period of 6 months (from January 2018 till June 2018), a single arm interventional study was conducted upon a convenience sample of 20 cases (11 males and 9 females) at an age range of 18-60 years old post complicated iatrogenic renal procedures.

The causative procedures were: renal biopsy in 9 cases (45\%), nephrostomy tube application in 5 cases (25\%), renal artery angioplasty in 2 cases (10\%), extracorporeal shock wave lithotripsy (ESWL) in 2 cases (10\%), percutaneous nephrolithotomy (PCNL) in 2 cases (10\%). 
These cases were referred for renal angiography +/- embolization, at the Interventional Radiology Unit of Ain Shams University Hospital using the "Toshiba Infinix INFX-8000V angiographic machine with the help of a digital subtraction angiography (DSA) software and a $360^{\circ}$ rotating tube.

\section{Methods:}

Preprocedural preparation: The study was approved by the ethical medical committee of Ain Shams University. Comprehensive medical history about the cases concomitant comorbidities and their presenting complaints. No significant comorbidities were recorded except for hypertension in cases with renal arterial stenosis. Informed written consents were taken preprocedural. Clinical examination, laboratory investigations and radiological assessment were carried out. Angiographic findings :The angiographically detected forms of renal arterial injury included: arteriovenous fistulas (AVFs), pseudoaneurysms, arterial extravasation with or without the development of a perinephric hematoma.

\section{Embolization procedure:}

Embolization Technique: It included the selective catheterization of the renal artery with a 5 French (F) Cobra catheter, a hydrophilic (Terumo) wire and an 18 gauge $(\mathrm{G})$ Seldinger needle using an over-the-wire (Seldinger) technique . Following the renal artery opacification with the exclusion of the presence of anatomical variants or accessory renal arteries, the bleeder was sealed by the appropriate embolic agent.

Embolic agents: The most efficient embolic agents used were the coils for proximal large bleeders, particles for small distant bleeders and gel foam for patients with abnormal bleeding profiles.

Postprocedural care and complications: The majority of the cases underwent the procedure with a minimal rate of complications. The developed complications included: post embolization syndrome, that presented with pain, fever and leukocytosis and were conservatively treated within the period of the post procedural hospital stay.

\section{Statistical analysis}

Data were collected, tabulated and statistically analyzed using a personal computer with (SPSS) version 22 program; (IBM SPSS Statistics for Windows, Version 22.0. Armonk, NY, USA: IBM Corp.).

\section{RESULTS}

The overall technical success rate in this study was $90 \%$ for RAE following iatrogenic renal arterial injury.

Clinical success was also $90 \%$ in terms of each of the following symptoms: abscence of recurrent haematuria with no $\mathrm{Hb}$ decrease or need for re embolization.

A statistically significant relation was found between the technical success and the angiographic embolizer used (Table (1)).

There was another statistically significant relation between the clinical and the technical success rates (Table (2)).

Table (1): The technical success rates variation with the usage of different angiographic embolizers.

\begin{tabular}{|l|c|c|c|c|c|c|c|}
\hline \multirow{2}{*}{$\begin{array}{c}\text { Angiographic } \\
\text { embolizer }\end{array}$} & \multicolumn{2}{|c|}{$\begin{array}{c}\text { Technical } \\
\text { success }\end{array}$} & $\begin{array}{c}\text { No } \\
\text { technical } \\
\text { success }\end{array}$ & \multirow{2}{*}{ Test value* } & P-value & Sig. \\
\cline { 2 - 5 } & No. & $\%$ & No. & $\%$ & & & \\
\hline Particles & 6 & $33.3 \%$ & 0 & $0.0 \%$ & & & \\
\hline Coils & 9 & $50.0 \%$ & 1 & $50.0 \%$ & 10.000 & 0.019 & $\mathrm{~S}$ \\
Gel foam & 3 & $16.7 \%$ & 0 & $0.0 \%$ & & & \\
Nal & 0 & $0.0 \%$ & 1 & $50.0 \%$ & & & \\
\hline
\end{tabular}

NS: Non significant; S: Significant; HS: Highly significant (:Chisquare test)

Table (2): The relation between the technical and clinical success rates

\begin{tabular}{|c|c|c|c|c|c|c|c|c|}
\hline \multicolumn{2}{|c|}{ Clinical success } & \multicolumn{2}{|c|}{$\begin{array}{c}\text { Technical } \\
\text { success }\end{array}$} & \multicolumn{2}{|c|}{$\begin{array}{c}\text { No } \\
\text { technical } \\
\text { success }\end{array}$} & \multirow[t]{2}{*}{ Test value* } & \multirow[t]{2}{*}{ P-value } & \multirow[t]{2}{*}{ Sig. } \\
\hline & & No. & $\%$ & No. & $\%$ & & & \\
\hline \multirow{2}{*}{$\begin{array}{l}\text { Haematuria (> } \\
3 \text { d) }\end{array}$} & No & 17 & $94.4 \%$ & 1 & $50.0 \%$ & \multirow{2}{*}{3.951} & \multirow{2}{*}{0.047} & \multirow{2}{*}{$\mathrm{S}$} \\
\hline & Yes & 1 & $5.6 \%$ & 1 & $50.0 \%$ & & & \\
\hline \multirow{2}{*}{$\mathrm{Hb}$ decrease } & No & 17 & $94.4 \%$ & 1 & $50.0 \%$ & \multirow{2}{*}{3.951} & \multirow{2}{*}{0.047} & \multirow{2}{*}{$\mathrm{S}$} \\
\hline & Yes & 1 & $5.6 \%$ & 1 & $50.0 \%$ & & & \\
\hline \multirow{2}{*}{$\begin{array}{l}\text { Need for } \\
\text { reembolization }\end{array}$} & No & 17 & $94.4 \%$ & 1 & $50.0 \%$ & \multirow{2}{*}{3.951} & \multirow{2}{*}{0.047} & \multirow{2}{*}{ S } \\
\hline & Yes & 1 & $5.6 \%$ & 1 & $50.0 \%$ & & & \\
\hline
\end{tabular}

\section{DISCUSSION}

The iatrogenic renal arterial injuries occur following renal surgical procedures or percutaneous renal interventions as in: biopsy or nephrostomy tube placement. The incidence of severe post operative renal hemorrhagic complications reaches up to $9.5 \%$. Prolonged bleeding leads to further complications including circulatory shock, acute kidney failure and disseminated intravascular coagulopathy ${ }^{(1)}$. 
The standard treatment options include conservative measures for asymptomatic/stable iatrogenically induced peri-renal hematomas, namely :close monitoring, bed rest and blood transfusion if needed. Surgical repair of renal arterial injury is usually difficult- especially in hemodynamically unstable patients- with a high risk of failure and possible subsequent nephrectomy ${ }^{(2)}$.

Renal angiography provides a high diagnostic value in the detection of iatrogenic renal vascular complications and possible therapeutic value if renal arterial embolization (RAE) is planned as an alternative to surgery. RAE is safe, minimally invasive with a better clinical outcome and a shortened hospital stay ${ }^{(3)}$.

Renal artery embolization is a selective technique based upon the concept of bleeders closure by utilizing an embolic agent delivered through an endovascular catheter. The choice of the embolic agent differs according to the form of the vascular compromise, the size and site of the bleeder ${ }^{(4)}$.

The different forms of iatrogenic renal arterial injuries that benefit from renal arterial embolization include: direct bleeding into the perirenal/subcapsular region with hematoma formation, bleeding into the pelvicalyceal system (arteriocalyceal fistulas), arteriovenous fistulas (AVFs) or pseudoaneurysms. Multiple injuries could exist concomitantly ${ }^{(5)}$.

Pseudo-aneurysms are unstable due to their high risk of rupture, thus requiring rapid intervention, preferably with metallic coils. Most AVFs are asymptomatic and resolve spontaneously within 24 months after diagnosis. Yet,if persistent gross hematuria, hemodynamic instability or renal function deterioration develops, AVFs are urgently closed using coils or gelfoam. When the AVF is very proximal to the renal artery,a stent graft can also be employed. Coils are generally used for proximal bleeders, particles for distal bleeders and gel foam for hemodynamically unstable patients ${ }^{(6)}$.

Although very rare, failure of RAE can be devastating and lead to total nephrectomy. Cases with more than two bleeding sites are identified risk factors for possible RAE failure ${ }^{(7)}$.

In our study, during a period of 6 months, a single arm interventional study was conducted upon a sample of 20 cases including both genders at an age range (18-60) years old post complicated iatrogenic renal procedures with technical and clinical success rates of $90 \%$ each. The most frequently used angiographic embolizers with the highest success rates were the coils. Most of the procedures were complication-free $(70 \%)$. The most frequently apparent complications were pain (10\%) followed by post embolization syndrome (5\%), both of which were adequately treated.

\section{Our results were comparable to the following studies:}

Jinga et al. conducted a study on 27 patients with iatrogenic renal arterial injuries achieving technical and clinical success rates of over $95 \%$, using coils and particles with equivalent success rates for $\mathrm{each}^{(\mathbf{8})}$. Moreover, Singh et al. conducted another study on 26 subjects with different causes of renal arterial injuries (traumatic injuries, iatrogenic injuries and spontaneous bleeding). It was reported that the technical and clinical success rates of renal artery embolization was $100 \%$, compared to the surgical alternative that had a success rate of $70-75 \%$.

Valecha et al. performed a study including 41 subjects exposed to iatrogenic renal arterial injuries and used particles only as angiographic embolizers. Both the technical and clinical success rates were $93 \%{ }^{(\mathbf{1 0})}$.

Zhaohui et al.carried out a study on 60 subjects who had been exposed to iatrogenic renal vascular injuries followed by renal arterial embolization, to detect the most frequently occuring complications. Post embolization syndrome was the most common (10\%), followed by infection (8\%) then recurrence of haematuria $(7 \%)^{(\mathbf{1 1 1})}$.

Kim et al.performed a clinical trial including 40 subjects, whose aim was to detect the most frequent radiologically detected forms of renal arterial injury to be treated by embolization. Pseudoaneurysms were found to be the most common form of arterial injury (40\%) followed by arterial extravasation $(35 \%)$. In our study, the frequency of occurence of the aforementioned symptoms was calculated, being equivocal between the pseudoaneurysms, AVFs and extravasation ${ }^{(\mathbf{1 2})}$.

Lobko et al. conducted a study to calculate the sensitivity of the imaging modalities in the detection of iatrogenic renal injuries requiring treatment. Angiography was 98\% sensitive to detect the vascular complications 
(pseudoaneurysms, A-V fistulas, extravasation), followed by the contrast enhanced CT (95\% in pseudoaneurysms and extravasation). Doppler ultrasonography is operator dependant ${ }^{(13)}$.

In our study, the frequency of usage of different imaging modalities for the initial detection was analyzed without a specific emphasis on the sensitivity of each modality for the detection of each form of arterial injury. Doppler ultrasonography was used in 9 cases $(45 \%)$ to initially detect a perinephric/subcapsular renal hematoma or the presence of free abdominopelvic fluid since it was cheap, fast, and available. Contrast enhanced CT was initially used in 4 cases (20\%) followed by angiography. Angiography was initially used only in case of emergency if the patient is hemodynamically unstable, or with the suspicion of a vascular fistuolous connection and embolization was planned in the same setting.

A meta analysis study was done by Muller and Rouvière ${ }^{(\mathbf{1 4})}$ to assess the efficacy and safety of RAE along with the effects of RAE on renal function and arterial blood pressure. The results indicated that RAE was technically successful in $98 \%$ of patients. Renal functions were followed for 4 months after RAE and blood pressures for 3 months. They concluded that RAE is not associated with a significant worsening of renal functions or increase in BP post procedural.The period of follow up in our study included only the period of postinterventional hospital stay ${ }^{(\mathbf{1 4})}$.

\section{CONCLUSION}

Iatrogenic renal arterial injuries pose significant morbidity and mortality. Surgical procedures mostly fail at the repair of the renal vascular injury that may reach up to nephrectomy with the loss of functioning renal parenchyma and a high postoperative morbidity and mortality rates.

Renal arterial embolization is a safe technique minimally invasive, and very efficient in the treatment of the aforementioned injuries as it helps to preserve as much renal tissue as possible with a lower rate of complications and with technical and clinical success rates of $90 \%$ each.
Improvements in the interventional tools including the development of various microcatheters and embolic agents have played a large role in the increasingly successful implementation of RAE for managing the renal vascular injuries with a high technical success rate.

\section{LIMITATIONS}

Limited sample size hindered the proper assessment of the efficacy of each embolic agent separately as regards its success rates and its subsequent complications. It also hindered the assessment of the success rate of the management of each form of renal vascular injuries treated by embolization.

The sensitivity of each imaging modality for the detection of each form of renal vascular injury was not assessed, hence it was not relatable to the technical or clinical success rates. Only the frequency of usage of each imaging modality in the initial assessment was calculated.

The technical and clinical success rates for embolization post renal blunt or penetrating trauma was not assessed since it was out of the scope of this study.

Unlike the American Association for the Surgery of Trauma (AAST) classification of the blunt renal trauma, no classification was found for the iatrogenic renal arterial injuries that could better delineate the lines of management for renal vascular iatrogenic injuies and hence emphasize the indications of renal arterial embolization versus surgery.

No comparison was done between the operative and the endovascular management of the renal arterial injuries in terms of the technical/clinical success rates and the complications rates.

The follow up of the complications post embolization only included the period of postinterventional hospital stay that was a few days up to a week, with no further follow up for the long term complications or side effects. 


\section{REFERENCES}

1. Froberg L, Helgstrand F, Clausen C, Steinmetz $J$ and Eckardt $H$ (2016): Mortality in trauma patients with active arterial bleeding managed by embolization or surgical packing: An observational cohort study of 66 patients. Journal of Emergencies, Trauma, and Shock, 9(3): 107.

2. Da Costa IA, Amend B, Stenzl A, Bedke J (2016): Contemporary management of acute kidney trauma. Journal of Acute Disease, 5(1): 29-36.

3. Doshi A, Manka MG, Pavlovich $\mathrm{C}$ and Auster M (2017): Hidden Renal Artery Pseudoaneurysm: The Need for Repeat Angiographic Intervention in a Symptomatic Patient. Urology Case Reports, 12: 54-55.

4. Loffroy R, Chevallier O, Gehin S, Midulla M, Berthod PE, Galland C and Falvo N (2017): Endovascular management of arterial injuries after blunt or iatrogenic renal trauma. Quantitative Imaging in Medicine and Surgery, 7(4): 434.

5. El Tayeb MM, Knoedler JJ, Krambeck AE, Paonessa JE, Mellon MJ, Lingeman JE (2015): Vascular complications after percutaneous nephrolithotomy: 10 years of experience. Urology, 85(4): 777-781.

6. Adrahtas D, Jasinski P, Koullias G, Fiorella $D$ and Tassiopoulos AK (2016): Endovascular treatment of a complex renal artery aneurysm using coils and the pipeline embolization device in a patient with a solitary kidney. Annuals of Vascular Surgery, 36: 291-299.

7. Khosla A and Wagner AA (2016): Robotic surgery of the kidney, bladder, and prostate. Surgical Clinics, 96(3): 615-636.

8. Jinga V, Dorobat B, Youssef S, Radavoi GD, Braticevici B, Filipoiu F, Balgradean $M$ (2013): Transarterial embolization of renal vascular lesions after percutaneous nephrolithotomy. Chirurgia, 108(4):521-539.

9. Singh G, Lopes DK, Jolly N (2016): Neuroendovascular Embolic Agent for Treatment of a Renal Arteriovenous Fistula. AIMS Med Sci., 3:96-102.

10. Valecha NK, Bagheri F, Hassani S, Sadi A, Souliman R (2017): Delayed Haematuria after Percutaneous Nephrolithotripsy and its Management. Med Sur Urol., 6(189):2.
11. Zhaohui H, Hanqi L, Xiongbing L, Caixia Z, Shawpong W, Guohua Z (2017): Analysis of repeated renal arteriography after percutaneous nephrolithotomy. Urolithiasis, 45(5):495-9.

12. Kim B, Han YM, Jin GY (2018): Transcatheter Arterial Embolization for Acute Arterial Extravasation with Hematoma Formation: Classified the Group as Cause and Their Clinical Outcomes. Journal of the Korean Society of Radiology,78(2): 453-445.

13. Lobko I, Mohabir A (2016): Renal Angiography and Embolization. In Interventional Urology, Springer, Cham. Pp: 283-298.

14. Muller A and Rouvière $O$ (2015): Renal artery embolization-indications, technical approaches and outcomes. Nature Reviews Nephrology, 11(5): 288. 